\title{
ENHANCING GROWTH, PRODUCTIVITY AND ESSENTIAL OIL PERCENTAGE OF THYMUS VULGARIS L. PLANT USING SEAWEEDS EXTRACT, CHITOSAN AND POTASSIUM SILICATE IN SANDY SOIL
}

\author{
A.A. Waly*; Y.M. Abd El-Fattah"; M.A.E. Hassan*; E.A.E. El-Ghadban** \\ and Alia S. Abd Alla** \\ * Floriculture, Medicinal \& Aromatic Plants, Hort. Dept., Agric. Fac., Suez Canal Univ., Egypt \\ ** Medicinal \& Aromatic plants Research Dept., Hort. Res. Inst., Agric. Res. Center, Egypt
}

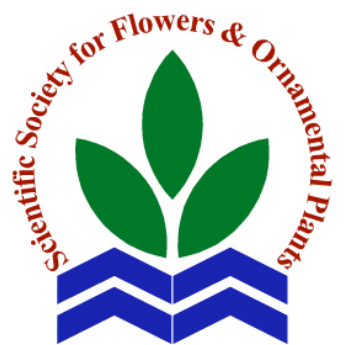

Scientific J. Flowers \& Ornamental Plants, 7(4):549-562 (2020).

Received: $12 / 11 / 2020$ Accepted: $4 / 12 / 2020$

ABSTRACT: The present study was carried out at the Experimental Farm, Horticulture Department, Faculty of Agriculture, Suez Canal University, Egypt during two successive seasons of 2017/2018 and $2018 / 2019$ to examine the effect of foliar application of seaweeds extract at 2, 4 and $6 \mathrm{ml} / 1$, chitosan at 2,4 and $6 \mathrm{ml} / 1$ and potassium silicate at 6,9 and $12 \mathrm{ml} / 1$ as well as the interaction between seaweeds extract and chitosan on growth, biochemical and essential oil characteristics of thyme plants grown in a sandy soil. These compounds were applied as foliar spray three times after 15 days from planting and at 15 days interval. The results showed that foliar applications of seaweeds extract at $6 \mathrm{ml} / 1$, chitosan at $6 \mathrm{ml} / 1$ and potassium silicate at $12 \mathrm{ml} / \mathrm{l}$ significantly affected all studied parameters compared with control. The results indicated that the different applied treatments increased the measured growth characteristics i.e. plant height, number of branches/plant, fresh and dry weights as well parallel increase of photosynthetic pigments. Also, constituents of N, P, K, total carbohydrates, percentage of essential oil and the GLC of essential oil of plant were existed in the two assigned seasons. In addition, the most effective combined treatments were seaweed at $6 \mathrm{ml} / 1+$ chitosan at $4 \mathrm{ml} / 1$ in this respect.

Key words: Medicinal and aromatic plants, thyme, Thymus vulgaris L., seaweed extract, chitosan, potassium silicate, essential oil.

\section{INTRODUCTION}

Thyme (Thymus vulgaris L.), locally known "zaatar", a member of the family Lamiaceae (Labiatae) is widely used in Egypt and Morocco folk medicine for its expectorant, antitussive, antibroncholytic antispasmodic, anthelmintic, carminative and diuretic properties. The aromatic and medicinal properties of the genus Thymus have made it one of the most popular plants all over the world. Numerous studies were conducted on medicinal plants to increase their productivity using different methods. Chitosan is a natural biopolymer modified from chitin, which can be transformed into chitosan by extracting the acetyl group and turn it into amino. Many investigators reported that using chitosan as foliar spray increased vegetative growth, yield and quality of different crops including Castro et al. (2016) on coriander; Mahdavi (2013) on isabgol (Plantago ovata Forsk) and Kim et al. (2005) on basil. Furthermore, chitosan has been recognized as a product to enhance crop production due to its bioactivities: biodegradability, growth stimulation and seed germination, increasing nutrient uptake, reducing oxidative stress, increasing 


\section{A.A. Waly et al.}

chlorophyll content, photosynthetic and chloroplast enlargement in the leaves, antifungal, antiviral and antibacterial properties (Hadrami et al., 2010 and Hadwiger, 2013). Seaweeds extract plays role as an activator of cell division, and gives rise to antioxidants levels for protection against adverse environmental conditions (Smirnoff, 1995). Nasiroleslami and Safaridolatabad (2014) reported that foliar application of seaweed increased vegetative growth of dill plants.

On the other hand, ppotassium is an important nutrient for plant meristematic growth and physiological functions, including regulation of water and gas exchange, protein synthesis, enzyme activation, and photosynthesis and carbohydrates translocation in plants. Potassium has favourable effects on metabolism of nucleic acids, proteins, vitamins, growth substances, energy transfer, phloem transport, cation-anion balance and enabling their ability to resist pests and diseases (Wang et al., 2013). Potassium silicate is a source of highly soluble silicon; it is used in agricultural production system primarily as a silicon fertilizer (Abou-Baker et al., 2011) on bean plant. Foliar spray with potassium silicate increased plant growth, chlorophyll content, $\mathrm{N}, \mathrm{P}$ and $\mathrm{K}$ contents in the leaves and yield and its components (Wand and Galletta, 1998) on strawberries.

Seaweeds are green, brown and red marine macroalgae. Extracts of brown seaweeds are widely used in horticulture crops largely for their plant growthpromoting effects and for their ameliorating effect on crop tolerance to abiotic stresses such as salinity, extreme temperatures, nutrient deficiency and drought. The chemical constituents of seaweed extract include complex polysaccharide, fatty acids, vitamins, phytohormones and mineral nutrients growth promoting hormones (IAA and IBA), cytokinins, trace elements ( $\mathrm{Fe}, \mathrm{Cu}$, $\mathrm{Zn}, \mathrm{Co}, \mathrm{Mo}, \mathrm{Mn}$, and Ni) and amino acids. (Battacharyyaet al., 2015).
Therefore, this study aimed to investigate the effect of seaweed extract, chitosan and potassium silicate on the growth, herb, essential oil yield and constituents as well as chemical compositions of thyme (Thymus vulgaris L.) plant.

\section{MATERIALS AND METHODS}

\section{Experimental design:}

The stem cuttings of thyme (Thymus vulgaris L.) were obtained from Experimental Farm of Medicinal \& Aromatic Plants Department, Horticultural Research Institute, Agriculture Research Center, Egypt. The plant materials are planted in the sandy field on December $26^{\text {th }}$ during the two successive seasons of 2017/2018 and 018/2019 at Experimental Farm, Faculty of Agriculture, Suez Canal University. The physical and chemical properties of the used water and experimental soil are shown in Tables (a and b). The plants were treated by spray extracts of seaweeds (2, 4 and $6 \mathrm{ml} / 1$ water), chitosan (2, 4 and $6 \mathrm{ml} / 1$ water) and potassium silicate (6, 9 and $12 \mathrm{ml} / 1$ water) as well as combinations between seaweeds and chitosan $(2: 2,2: 4$, and $2: 6 \mathrm{ml} / 1,4: 2,4: 4$, and $4: 6 \mathrm{ml} / 1$, and $6: 2,6: 4$, and $6: 6 \mathrm{ml} / \mathrm{l})$. The plants were sprayed with the tested treatments three times with 15 days interval between spraying and the next, the first spray was done 15 days after planting. Seaweed extract coined as Oligo-X was used in this study. It is a mixture of Ascophyllum spp., Laminaria spp., Sargassum spp. and Fucus spp. It was obtained from Union for Agricultural Development Company, Egypt; the chemical constituents are shown in Table (c).

\section{Morphological attributes:}

Data of the morphological traits were recorded in each cutting separately for estimating the variability in effect of the different treatments on plant growth. These data included plant height $(\mathrm{cm})$, number of branches/plant, fresh and dry weights of plant (g/plant). 
Table a. Chemical and physical properties of the experimental soil.

\begin{tabular}{|c|c|c|c|c|c|c|c|c|}
\hline \multirow{2}{*}{ 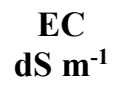 } & \multicolumn{4}{|c|}{ Anions (ppm) } & \multicolumn{4}{|c|}{ Cations (ppm) } \\
\hline & $\mathrm{CO}_{3}^{-}$ & $\mathrm{HCO}_{3}^{-}$ & $\mathbf{C L}^{-}$ & $\mathrm{SO}_{4}^{--}$ & $\mathbf{C a}^{++}$ & $\mathbf{M g}^{++}$ & $\mathbf{N a}^{+}$ & $\mathbf{K}^{+}$ \\
\hline 0.58 & -- & 2.12 & 2.54 & 1.67 & 4.60 & 0.83 & 0.82 & 0.08 \\
\hline \multirow{2}{*}{ pH } & \multicolumn{4}{|c|}{ Macro-elements (mg/1000g) } & \multicolumn{4}{|c|}{ Micro-elements (mg/1000g) } \\
\hline & $\mathbf{N}$ & & & $\mathbf{P}$ & $\mathbf{C u}^{++}$ & $\mathrm{Fe}^{++}$ & $\mathbf{M n}^{++}$ & $\mathbf{Z n}^{++}$ \\
\hline 8 & 53.0 & & & 0.90 & 0.90 & 2.20 & 1.54 & 0.59 \\
\hline
\end{tabular}

Table b. Chemical and physical properties of used water.

\begin{tabular}{|c|c|c|c|c|c|c|c|c|}
\hline \multirow{2}{*}{$\begin{array}{c}\mathbf{E C} \\
\mathrm{dS} \mathbf{m}^{-1}\end{array}$} & \multicolumn{4}{|c|}{ Anions (ppm) } & \multicolumn{4}{|c|}{ Cations (ppm) } \\
\hline & $\mathrm{CO}_{3}^{-}$ & $\mathrm{HCO}_{3}{ }^{-}$ & $\mathbf{C L}^{-}$ & $\mathrm{SO}_{4}^{-}$ & $\mathrm{Ca}^{++}$ & $\mathbf{M g}^{++}$ & $\mathbf{N a}^{+}$ & $\mathbf{K}^{+}$ \\
\hline 0.40 & -- & 1.42 & 0.85 & 2.00 & 2.82 & 1.08 & 0.26 & 0.11 \\
\hline \multicolumn{9}{|c|}{ Elements mg/l } \\
\hline pH & $\mathbf{N H}_{4}{ }^{+}$ & $\mathrm{NO}_{3}^{-}$ & B & $\mathbf{C U}$ & $\mathrm{Fe}$ & Mn & $\mathbf{P}$ & $\mathbf{Z n}$ \\
\hline 7.10 & 1.05 & 1.75 & 0.30 & 0.20 & 0.20 & 0.011 & 1.50 & 0.30 \\
\hline
\end{tabular}

Table c. Chemical constituents of Oligo-X seaweed extract.

\begin{tabular}{|c|c|c|c|c|c|c|c|c|c|c|c|}
\hline \multirow[b]{2}{*}{ 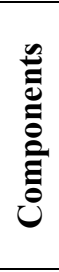 } & \multirow[b]{2}{*}{ 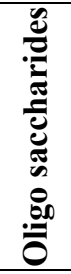 } & \multirow[b]{2}{*}{ 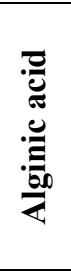 } & \multirow[b]{2}{*}{ 漓 } & \multirow[b]{2}{*}{ 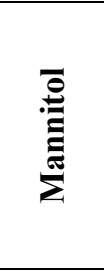 } & \multirow[b]{2}{*}{ 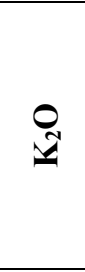 } & \multirow[b]{2}{*}{ 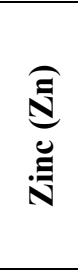 } & \multirow[b]{2}{*}{ 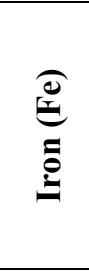 } & \multirow[b]{2}{*}{ 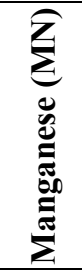 } & \multicolumn{3}{|c|}{ Growth regulators } \\
\hline & & & & & & & & & 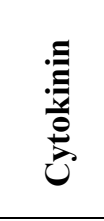 & 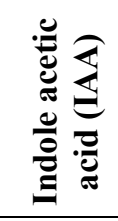 & 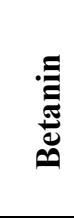 \\
\hline$\%$ & 3 & 5 & 0.003 & 0.001 & 4.71 & 0.13 & 0.12 & 0.10 & 0.001 & 0.0002 & 0.02 \\
\hline
\end{tabular}

The first cut was done on April $1^{\text {st }}$ and the second cut was done on $15^{\text {th }}$ June of both seasons. The plants were about $5 \mathrm{~cm}$ above the ground leaving a lateral branch to renew the plant growth.

\section{Chemical analysis:}

- The contents of chlorophylls (a, b) and carotenoids were determined in the fresh leaves as described by Mazumdar and Majumder (2003). Total chlorophylls and carotenoids were determined (mg/g f.w.) according to Wettestein (1957).

- Contents of leaf minerals were estimated according to Piper (1947).

- Percentage of nitrogen was determined by using the micro-Kjeldhle method as described by Jackson (1967).

- Phosphorus percentage was calorimetrically determined according to Murphy and Reilly (1962).
- Potassium percentage was determined using the atomic absorption spectrophotometer (3300) according to Wilde et al. (1985).

- Total carbohydrates \% of the dry weight were determined according to Herbert et al. (1971).

- Essential oil percentage (EO) was determined according to British Pharmacopoeia (2000).

- The essential oil content was analyzed using gas liquid chromatography (GLC) to determine the main constituents of the essential oil according to Hoftman (1967). GLC was carried out as described by Guenther and Joseph (1978).

\section{Statistical analysis:}

The experimental design was complete randomized blocks design included 19 treatments + control treatment with three replicates. The replicate area was $1.5 \times 1.5 \mathrm{~m}$. 


\section{A.A. Waly et al.}

Each replicate was included 16 plant; the distance between plants was $35 \mathrm{~cm}$. Data were subjected to analysis of variance (ANOVA) by the general linear models (LMS) procedure using (CoStat) statistical analysis system. Mean comparisons were performed using the least significant differences (L.S.D) method at significance level of 5\% according to Gomez and Gomez (1984).

\section{RESULTS AND DISCUSSIONS}

Vegetative growth and herb yield:

Effect of seaweed extract, chitosan and potassium silicate:

Thyme plants foliar sprayed with seaweed extract at different levels increased the vegetative growth characteristics compared to control. Plant height $(\mathrm{cm})$, number of branches/plant, herb fresh and dry weights (g/plant) were increased with increasing sprayed foliar concentration of seaweed extract (4 and $6 \mathrm{ml} / \mathrm{l}$ ) and potassium silicate up to $12 \mathrm{ml} / \mathrm{l}$ as well as chitosan up to (4 and $6 \mathrm{ml} / \mathrm{l}$ ) in both seasons and for the two cuts compared with control plants, as presented in Table (1), nevertheless the control treatment gave the lowest value in the two experimental seasons.

With respect to vegetative fresh weight per plant, results shown in Table (1) clear that seaweed extract at $6 \mathrm{ml} / \mathrm{l}$ as a foliar spray showed the highest values; on the other hand, control plants recorded the lowest values in the two tested seasons. Such results are in the same line with those of Norrie and Keathley (2006), Hussein et al (2011), and Delucia and Vecchietti (2012). This increment in the vegetative fresh weight/plant may be due to the positive effect of seaweed extract on increasing most vegetative growth characters i.e., plant height, number of shoots and flower stalk length that seaweed extract contains many growth regulators, macro and micro nutrients

Table 1. Effect of seaweed extract, chitosan and potassium silicate on vegetative growth of Thymus vulgaris L. for the two cuts during 2017/2018 and 2018/2019 seasons.

\begin{tabular}{|c|c|c|c|c|c|c|c|c|}
\hline \multirow{2}{*}{ Treatments } & \multicolumn{2}{|c|}{ Plant height (cm) } & \multicolumn{2}{|c|}{ No. of branches/plant } & \multicolumn{2}{|c|}{ F.W. (g)/plant } & \multicolumn{2}{|c|}{ D.W. (g)/plant } \\
\hline & $1^{\text {st }}$ cut & $2^{\text {nd }}$ cut & $1^{\text {st }}$ cut & $2^{\text {nd }}$ cut & $1^{\text {st }}$ cut & $2^{\text {nd }}$ cut & $1^{\text {st }}$ cut & $2^{\text {nd }} \mathrm{cu}$ \\
\hline & \multicolumn{8}{|c|}{ First season $(2017 / 2018)$} \\
\hline Control & 7.33 & 11.3 & 24.3 & 25.6 & 9.86 & 15.81 & 5.10 & 7.26 \\
\hline Sw $2 \mathrm{ml} / \mathrm{l}$ & 12.3 & 14.7 & 44.3 & 51.3 & 21.26 & 25.89 & 7.83 & 10.63 \\
\hline Sw $4 \mathrm{ml} / \mathrm{l}$ & 14.3 & 15.4 & 57.0 & 62.3 & 27.71 & 29.46 & 8.66 & 12.30 \\
\hline Sw $6 \mathrm{ml} / \mathrm{l}$ & 17.0 & 19.6 & 86.3 & 85.3 & 34.80 & 35.57 & 13.83 & 15.30 \\
\hline Ch $2 \mathrm{ml} / \mathrm{l}$ & 11.6 & 13.5 & 35.3 & 48.6 & 13.64 & 25.56 & 7.20 & 9.56 \\
\hline Ch $4 \mathrm{ml} / \mathrm{l}$ & 13.0 & 14.3 & 53.3 & 53.3 & 24.55 & 27.31 & 9.56 & 11.53 \\
\hline Ch $6 \mathrm{ml} / \mathrm{l}$ & 14.0 & 16.8 & 74.3 & 68.3 & 30.39 & 33.22 & 11.10 & 13.33 \\
\hline Ksi 6 ml/l & 12.0 & 13.6 & 40.6 & 47.0 & 18.87 & 23.50 & 6.86 & 7.63 \\
\hline Ksi 9 ml/l & 13.3 & 14.3 & 53.3 & 61.0 & 30.39 & 28.98 & 10.16 & 13.13 \\
\hline Ksi 12 ml/l & 15.2 & 18.5 & 85.3 & 80.6 & 32.99 & 34.54 & 13.13 & 14.23 \\
\hline \multirow[t]{2}{*}{ L.S.D. at 5\% } & 2.33 & 2.53 & 9.94 & 12.92 & 6.67 & 3.19 & 2.44 & 1.76 \\
\hline & \multicolumn{8}{|c|}{ Second season $(2018 / 2019)$} \\
\hline Control & 12.3 & 14.6 & 31.3 & 46.3 & 28.41 & 34.41 & 12.46 & 13.7 \\
\hline Sw $2 \mathrm{ml} / \mathrm{l}$ & 20.3 & 20.3 & 80.3 & 98.6 & 35.16 & 43.19 & 18.66 & 21.33 \\
\hline Sw $4 \mathrm{ml} / \mathrm{l}$ & 21.3 & 23.6 & 107.0 & 120.3 & 44.65 & 56.73 & 21.96 & 25.23 \\
\hline Sw $6 \mathrm{ml} / \mathrm{l}$ & 23.6 & 26.0 & 121.6 & 131.6 & 57.43 & 63.14 & 22.66 & 34.23 \\
\hline Ch $2 \mathrm{ml} / \mathrm{l}$ & 18.3 & 17.3 & 63.30 & 65.30 & 32.69 & 38.76 & 15.10 & 15.00 \\
\hline Ch $4 \mathrm{ml} / \mathrm{l}$ & 20.0 & 22.6 & 91.30 & 99.00 & 37.71 & 52.58 & 19.70 & 19.96 \\
\hline Ch $6 \mathrm{ml} / \mathrm{l}$ & 21.8 & 23.6 & 95.30 & 118.3 & 41.25 & 62.12 & 20.73 & 30.96 \\
\hline Ksi 6 ml/l & 19.0 & 20.3 & 75.30 & 80.6 & 36.36 & 44.46 & 14.33 & 19.66 \\
\hline Ksi 9 ml/l & 21.0 & 22.3 & 97.30 & 110.0 & 38.29 & 53.37 & 19.13 & 20.33 \\
\hline $\mathrm{Ksi} 12 \mathrm{ml} / \mathrm{l}$ & 22.3 & 24.0 & 119.6 & 129.3 & 55.43 & 63.71 & 21.96 & 31.66 \\
\hline L.S.D. at 5\% & 2.42 & 2.21 & 11.06 & 9.51 & 7.42 & 7.23 & 3.84 & 3.59 \\
\hline
\end{tabular}

Sw: seaweed extract, $\mathrm{Ch}$ : chitosan, Ksi: potassium silicate 
as reported by Shaaban (2011) on wheat, and Ordog et al (2004) and Jensen (2004).

\section{Effect of combination between seaweed extract and chitosan:}

Foliar spraying of chitosan and seaweeds extract significantly affected all growth characters [i.e. plant height $(\mathrm{cm})$, number of branches/plant, herb fresh and dry weights (g/plant) in the two cuts of both seasons of the study (Table, 2). However, previous studies proved that seaweeds and/or chitosan can, directly or indirectly, influence the physiological activities of the plants (Kamal and Ghanem, 2011). Moreover, Kim et al. (2005) reported that chitosan has been shown to stimulate growth of sweet basil plants and Saif Eldeen et al. (2014) on globe artichoke plants attained similar result.
Essential oil production:

1. Essential oil percentage:

a. Effect of seaweed extract, chitosan and potassium silicate:

Data presented in Table (3) showed a significant difference in the two seasons, among potassium silicate, seaweed extract and chitosan treatments. The highest values of essential oil $\%$ were due to seaweed extract treatment, while potassium silicate and chitosan gave intermediate values. On the other hand, the least values of essential oil \% were given by control treatment. It was observed that applying each of seaweed extract, potassium silicate or chitosan at the high concentration gave better results than those obtained from the low concentration. The highest values of the essential oil percentage ranged between $0.36-0.37$ and $0.38-0.40 \%$ compared to the control 0.23 ,

Table 2. Effect of combination treatments between seaweed extract and chitosan on vegetative growth of Thymus vulgaris $L$. for the two cuts during 2017/2018 and 2018/2019 seasons.

\begin{tabular}{|c|c|c|c|c|c|c|c|c|}
\hline \multirow{2}{*}{ Treatments } & \multicolumn{2}{|c|}{$\begin{array}{l}\text { Plant height } \\
\text { (cm) }\end{array}$} & \multicolumn{2}{|c|}{$\begin{array}{c}\text { No. of } \\
\text { branches/plant }\end{array}$} & \multicolumn{2}{|c|}{ F. W. (g) /plant } & \multicolumn{2}{|c|}{ D. W. (g) /plant } \\
\hline & $1^{\text {st }}$ cut & $2^{\text {nd }}$ cut & $1^{\text {st }}$ cut & $2^{\text {nd }}$ cut & $1^{\text {st }}$ cut & $2^{\text {nd }}$ cut & $1^{\text {st }}$ cut & $2^{\text {nd }}$ cut \\
\hline & \multicolumn{8}{|c|}{ First season $(2017 / 2018)$} \\
\hline Control & 7.33 & 11.3 & 24.3 & 25.6 & 9.86 & 15.81 & 5.10 & 7.26 \\
\hline $\mathrm{Sw} 2 \mathrm{ml} / \mathrm{l}+\mathrm{Ch} 2 \mathrm{ml} / \mathrm{l}$ & 14.6 & 15.3 & 69.3 & 80.0 & 35.18 & 35.50 & 12.56 & 14.40 \\
\hline $\mathrm{Sw} 2 \mathrm{ml} / \mathrm{l}+\mathrm{Ch} 4 \mathrm{ml} / \mathrm{l}$ & 14.0 & 16.1 & 114.0 & 116.0 & 36.06 & 36.08 & 15.36 & 15.20 \\
\hline $\mathrm{Sw} 2 \mathrm{ml} / \mathrm{l}+\mathrm{Ch} 6 \mathrm{ml} / \mathrm{l}$ & 16.3 & 17.0 & 152.3 & 167.3 & 38.14 & 39.48 & 13.33 & 15.33 \\
\hline $\mathrm{Sw} 4 \mathrm{ml} / \mathrm{l}+\mathrm{Ch} 2 \mathrm{ml} / \mathrm{l}$ & 17.6 & 18.3 & 176.3 & 188.3 & 42.26 & 39.48 & 14.31 & 16.00 \\
\hline $\mathrm{Sw} 4 \mathrm{ml} / \mathrm{l}+\mathrm{Ch} 4 \mathrm{ml} / \mathrm{l}$ & 18.3 & 19.0 & 192.0 & 196.0 & 49.12 & 43.19 & 15.41 & 19.00 \\
\hline Sw 4 ml/l + Ch 6 ml/l & 19.0 & 20.1 & 194.0 & 199.6 & 50.09 & 47.97 & 16.36 & 19.00 \\
\hline Sw $6 \mathrm{ml} / \mathrm{l}+\mathrm{Ch} 2 \mathrm{ml} / \mathrm{l}$ & 16.6 & 17.6 & 188.6 & 191.0 & 43.13 & 46.82 & 14.73 & 17.13 \\
\hline $\mathrm{Sw} 6 \mathrm{ml} / \mathrm{l}+\mathrm{Ch} 4 \mathrm{ml} / \mathrm{l}$ & 21.3 & 24.6 & 209.0 & 215.0 & 52.14 & 53.14 & 19.46 & 21.36 \\
\hline $\mathrm{Sw} 6 \mathrm{ml} / \mathrm{l}+\mathrm{Ch} 6 \mathrm{ml} / \mathrm{l}$ & 20.0 & 21.6 & 206.0 & 214.0 & 51.38 & 52.14 & 18.40 & 20.00 \\
\hline \multirow[t]{2}{*}{ L.S.D. at 5\% } & 2.62 & 2.77 & 8.94 & 8.68 & 5.83 & 6.09 & 1.85 & 2.23 \\
\hline & \multicolumn{8}{|c|}{ Second season $(2018 / 2019)$} \\
\hline Control & 12.3 & 14.6 & 31.3 & 46.3 & 28.41 & 34.41 & 12.46 & 13.7 \\
\hline $\mathrm{Sw} 2 \mathrm{ml} / \mathrm{l}+\mathrm{Ch} 2 \mathrm{ml} / \mathrm{l}$ & 21.3 & 24.3 & 147.0 & 165.6 & 48.18 & 68.00 & 24.16 & 33.83 \\
\hline $\mathrm{Sw} 2 \mathrm{ml} / \mathrm{l}+\mathrm{Ch} 4 \mathrm{ml} / \mathrm{l}$ & 23.5 & 24.3 & 175.6 & 175.3 & 49.47 & 75.62 & 23.76 & 36.03 \\
\hline $\mathrm{Sw} 2 \mathrm{ml} / \mathrm{l}+\mathrm{Ch} 6 \mathrm{ml} / \mathrm{l}$ & 23.3 & 25.6 & 181.6 & 184.6 & 60.52 & 85.96 & 25.43 & 38.43 \\
\hline $\mathrm{Sw} 4 \mathrm{ml} / \mathrm{l}+\mathrm{Ch} 2 \mathrm{ml} / \mathrm{l}$ & 24.0 & 26.3 & 183.0 & 197.3 & 66.84 & 91.15 & 25.66 & 49.76 \\
\hline $\mathrm{Sw} 4 \mathrm{ml} / \mathrm{l}+\mathrm{Ch} 4 \mathrm{ml} / \mathrm{l}$ & 25.0 & 27.6 & 207.0 & 217.3 & 69.96 & 108.0 & 30.63 & 73.66 \\
\hline Sw $4 \mathrm{ml} / \mathrm{l}+\mathrm{Ch} 6 \mathrm{ml} / \mathrm{l}$ & 26.0 & 31.6 & 212.0 & 222.6 & 76.58 & 114.0 & 32.33 & 75.50 \\
\hline $\mathrm{Sw} 6 \mathrm{ml} / \mathrm{l}+\mathrm{Ch} 2 \mathrm{ml} / \mathrm{l}$ & 24.0 & 26.6 & 187.3 & 211.6 & 68.01 & 97.00 & 28.33 & 55.66 \\
\hline Sw $6 \mathrm{ml} / \mathrm{l}+\mathrm{Ch} 4 \mathrm{ml} / \mathrm{l}$ & 27.0 & 32.0 & 247.6 & 258.0 & 80.21 & 135.4 & 39.00 & 78.66 \\
\hline Sw $6 \mathrm{ml} / \mathrm{l}+\mathrm{Ch} 6 \mathrm{ml} / \mathrm{l}$ & 26.3 & 31.0 & 219.3 & 225.0 & 73.65 & 121.9 & 34.50 & 76.50 \\
\hline L.S.D. at 5\% & 2.89 & 2.53 & 19.13 & 18.10 & 13.64 & 5.89 & 2.68 & 5.28 \\
\hline
\end{tabular}

Sw: seaweed extract, $\mathrm{Ch}$ : chitosan 
Table 3. Effect of seaweeds, chitosan and potassium silicate on essential oil percentage of Thymus vulgaris $L$. for two cuts during $2017 / 2018$ and 2018/2019 seasons.

\begin{tabular}{|c|c|c|c|c|}
\hline \multirow{2}{*}{ Treatments } & \multicolumn{4}{|c|}{ Essential oil (\%) } \\
\hline & $1^{\text {st }}$ cut & $2^{\text {nd }}$ cut & $1^{\text {st }}$ cut & $2^{\text {nd }} \mathrm{cu}$ \\
\hline & \multicolumn{2}{|c|}{ First season (2017/2018) } & \multicolumn{2}{|c|}{ Second season $(2018 / 2019)$} \\
\hline Control & 0.23 & 0.24 & 0.22 & 0.28 \\
\hline Sw $2 \mathrm{ml} / \mathrm{l}$ & 0.27 & 0.30 & 0.32 & 0.35 \\
\hline Sw $4 \mathrm{ml} / \mathrm{l}$ & 0.33 & 0.35 & 0.36 & 0.38 \\
\hline Sw $6 \mathrm{ml} / \mathrm{l}$ & 0.36 & 0.37 & 0.38 & 0.40 \\
\hline Ch 2 ml/l & 0.26 & 0.28 & 0.31 & 0.33 \\
\hline Ch 4 ml/l & 0.29 & 0.30 & 0.34 & 0.35 \\
\hline Ch 6 ml/l & 0.30 & 0.32 & 0.35 & 0.37 \\
\hline Ksi 6 ml/l & 0.29 & 0.30 & 0.32 & 0.34 \\
\hline Ksi 9 ml/l & 0.32 & 0.33 & 0.35 & 0.37 \\
\hline Ksi $12 \mathrm{ml} / \mathrm{l}$ & 0.34 & 0.35 & 0.36 & 0.39 \\
\hline L.S.D. at 5\% & 0.112 & 0.135 & 0.169 & 0.127 \\
\hline
\end{tabular}

Sw: seaweed extract, Ch: chitosan, Ksi: potassium silicate

$0.24-0.22$ and $0.28 \%$ in the first and second cuts in both seasons of study, respectively.

Similar results concerning seaweed extract fertilization were pointed out by Gharib et al. (2014) on rosemary; Salama and Yousef (2015) on Ocimum sanctum L.; Atteya and Amer (2018) on Hibiscus sabdariffa; El-leithy et al. (2019) on Plectuanthus amboinicus. Also, potassium silicate effect was found by Ezz El-Din et al. (2010) on caraway; Khalid (2014) and Shabana et al. (2016) on Salvia officinalis; and concerning chitosan was found by Helaly et al. (2018) on rosemary (Rosmarinus officinalis L.).

\section{b. Effect of combination between seaweed extract and chitosan:}

Data presented in Table (4) showed that spraying combination between seaweeds 6 $\mathrm{ml} / \mathrm{l}+$ chitosan $4 \mathrm{ml} / 1$ treatments significantly produced the highest values of essential oil percentage. oil percentage recorded $0.52,0.55 \%$ for the first and second cuts in the first season, respectively and 0.55 , $0.58 \%$ for the first and second cuts for the second season, orderly. While, the lowest essential oil percentage were recorded $(0.23$, $0.22)$ and $(0.24,0.28)$ resulted from the control treatment at the first cut and second one during both seasons of study successfully.

\section{Essential oil components:}

The essential oil ingredient (\%) of Thymus Vulgaris L. plant as affected by the combination between foliar application of seaweed extract and chitosan in the first cut at the second season were shown in Table (5) and Figure (1). The components were $\alpha-$ pinene, camphene, $\alpha$-terpinene, $p$-cymene, limonene, Y-terpinene, terpineolene, borneol, thymol and carvacrol. The spraying combination between seaweeds at $6 \mathrm{ml} / 1+$ chitosan at $4 \mathrm{ml} / \mathrm{l}$ treatment raised oil contents from thymol, p-cymene and $\alpha-$ terpinene to the highest values of $24.47,19.07$ and $17.47 \%$, respectively. The least total component resulted from untreated plants $(83.54 \%)$ and thymol, p-cymene and $\alpha$-Terpinene recorded 10.84, 12.46 and 18.31 , orderly. These results were in harmony with those of Kim et al. (2005) on Ocimum basilicum L and Bistgani et al. (2016) on Thymus daenensis.

\section{Chemical constituents:}

\section{Photosynthetic pigments content:}

\section{a. Effect of seaweed extract, chitosan and potassium silicate:}

Data illustrated in Table (6) showed that all concentrations of seaweeds extract, potassium silicate and chitosan significantly increased the photosynthetic pigments of chlorophyll a, b, total carotenoids and total 
Table 4. Effect of combination treatments between seaweed extract and chitosan on essential oil percentage of Thymus vulgaris $\mathrm{L}$. for two cuts during 2017/2018 and 2018 /2019 seasons.

\begin{tabular}{|c|c|c|c|c|}
\hline \multirow{2}{*}{ Treatments } & \multicolumn{4}{|c|}{ Essential oil (\%) } \\
\hline & $1^{\text {st }}$ cut & $2^{\text {nd }}$ cut & $1^{\text {st }}$ cut & $2^{\text {nd }}$ cut \\
\hline & \multicolumn{2}{|c|}{ First season $(2017 / 2018)$} & \multicolumn{2}{|c|}{ Second season $(2018 / 2019)$} \\
\hline Control & 0.23 & 0.24 & 0.22 & 0.28 \\
\hline $\mathrm{Sw} 2 \mathrm{ml} / \mathrm{l}+\mathrm{Ch} 2 \mathrm{ml} / \mathrm{l}$ & 0.30 & 0.31 & 0.33 & 0.35 \\
\hline $\mathrm{Sw} 2 \mathrm{ml} / \mathrm{l}+\mathrm{Ch} 4 \mathrm{ml} / \mathrm{l}$ & 0.34 & 0.36 & 0.32 & 0.38 \\
\hline Sw $2 \mathrm{ml} / \mathrm{l}+\mathrm{Ch} 6 \mathrm{ml} / \mathrm{l}$ & 0.35 & 0.35 & 0.37 & 0.39 \\
\hline Sw $4 \mathrm{ml} / \mathrm{l}+\mathrm{Ch} 2 \mathrm{ml} / \mathrm{l}$ & 0.39 & 0.40 & 0.41 & 0.46 \\
\hline Sw $4 \mathrm{ml} / \mathrm{l}+\mathrm{Ch} 4 \mathrm{ml} / \mathrm{l}$ & 0.47 & 0.49 & 0.46 & 0.53 \\
\hline Sw $4 \mathrm{ml} / \mathrm{l}+\mathrm{Ch} 6 \mathrm{ml} / \mathrm{l}$ & 0.48 & 0.48 & 0.50 & 0.54 \\
\hline Sw $6 \mathrm{ml} / \mathrm{l}+\mathrm{Ch} 2 \mathrm{ml} / \mathrm{l}$ & 0.45 & 0.46 & 0.49 & 0.51 \\
\hline Sw $6 \mathrm{ml} / \mathrm{l}+\mathrm{Ch} 4 \mathrm{ml} / \mathrm{l}$ & 0.52 & 0.55 & 0.55 & 0.58 \\
\hline Sw $6 \mathrm{ml} / \mathrm{l}+\mathrm{Ch} 6 \mathrm{ml} / \mathrm{l}$ & 0.50 & 0.53 & 0.54 & 0.56 \\
\hline L.S.D. 5\% & 0.271 & 0.292 & 0.324 & 0.280 \\
\hline
\end{tabular}

Sw: Seaweed, Ch: Chitosan

Table 5. Essential oil components (\%) of Thymus vulgaris L. plant as affected by the combination between foliar extract seaweed and chitosan in the $2^{\text {nd }}$ cut at the second season (2018/2019).

\begin{tabular}{|c|c|c|c|c|c|c|c|c|c|c|c|c|}
\hline \multirow{3}{*}{ Components (\%) } & \multicolumn{12}{|c|}{ Treatments } \\
\hline & \multicolumn{4}{|c|}{ Sw $2 \mathrm{ml} / \mathrm{l}$} & \multicolumn{4}{|c|}{$\mathrm{Sw} 4 \mathrm{ml} / \mathrm{l}$} & \multicolumn{4}{|c|}{ Sw $6 \mathrm{ml} / \mathrm{l}$} \\
\hline & CON & $\begin{array}{c}\mathrm{Ch} \\
2 \mathrm{ml} / \mathrm{l} \\
\end{array}$ & $\begin{array}{c}\text { Ch } \\
4 \mathrm{ml} / \mathrm{l} \\
\end{array}$ & $\begin{array}{c}\mathrm{Ch} \\
6 \mathrm{ml} / \mathrm{l} \\
\end{array}$ & CON & $\begin{array}{c}\mathrm{Ch} \\
2 \mathrm{ml} / \mathrm{l} \\
\end{array}$ & $\begin{array}{c}\mathrm{Ch} \\
4 \mathrm{ml} / \mathrm{I} \\
\end{array}$ & $\begin{array}{c}\text { Ch } \\
6 \mathrm{ml} / \mathrm{l} \\
\end{array}$ & CON & $\begin{array}{c}\mathrm{Ch} \\
2 \mathrm{ml} / \mathrm{l} \\
\end{array}$ & $\begin{array}{c}\mathrm{Ch} \\
4 \mathrm{ml} / \mathrm{l} \\
\end{array}$ & $\begin{array}{c}\mathrm{Ch} \\
6 \mathrm{ml} / \mathrm{l} \\
\end{array}$ \\
\hline . & & 4.93 & 4.85 & 4.94 & 3.32 & 4.37 & 4.39 & 4.39 & 4.04 & 4.39 & 4.50 & 4.42 \\
\hline Camphene & 13.10 & 8.21 & 9.78 & 12.06 & 9.52 & 13.20 & 9.93 & 10.34 & 5.92 & 9.93 & 4.06 & 8.51 \\
\hline$\alpha$-Terpinene & 18.31 & 17.47 & 15.54 & 18.75 & 11.78 & 15.98 & 16.08 & 14.31 & 12.07 & 16.08 & 17.74 & 16.68 \\
\hline p-Cymene & 12.46 & 19.63 & 17.27 & 22.96 & 14.93 & 2.77 & 17.64 & 17.27 & 16.73 & 17.64 & 19.07 & 18.08 \\
\hline Limonene & 2.02 & 2.80 & 2.19 & 1.75 & 1.91 & 7.02 & 1.97 & 2.19 & 2.47 & 1.97 & 2.26 & 1.98 \\
\hline Y -Terpinene & 2.87 & 6.55 & 7.24 & 2.98 & 5.98 & 4.26 & 6.90 & 7.32 & 6.97 & 6.90 & 7.99 & 7.41 \\
\hline Terpineolene & 11.53 & 9.10 & 7.68 & 13.58 & 8.07 & 6.39 & 9.49 & 10.17 & 9.30 & 9.49 & 9.83 & 9.31 \\
\hline Borneol & 6.39 & 6.71 & 6.74 & 6.56 & 6.83 & 10.79 & 6.73 & 7.24 & 7.97 & 6.73 & 7.57 & 6.93 \\
\hline Thymol & 10.84 & 15.69 & 22.26 & 11.11 & 18.24 & 21.93 & 21.94 & 21.08 & 22.71 & 21.94 & 24.47 & 23.76 \\
\hline Carvacrol & 0.75 & 1.40 & 1.39 & 0.77 & 5.71 & 2.63 & 1.43 & 1.95 & 2.25 & 1.43 & 1.53 & 1.45 \\
\hline Total components & 83.54 & 92.49 & 94.94 & 95.46 & 86.29 & 89.34 & 96.50 & 96.26 & 90.43 & 96.50 & 99.42 & 98.53 \\
\hline
\end{tabular}

CON: control, Sw: seaweed, Ch: chitosan 
A.A. Waly et al.

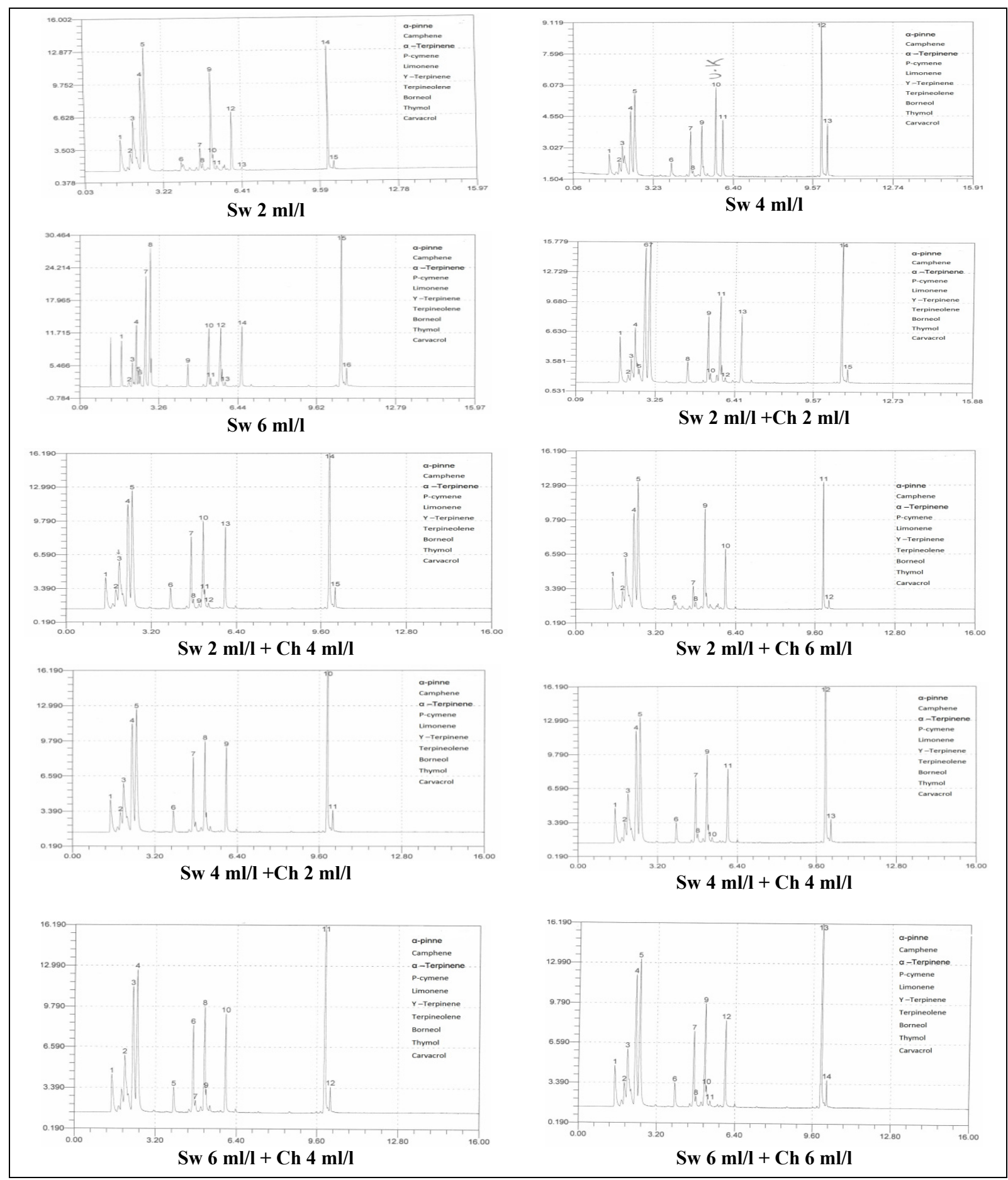

Fig. 1. Essential oil components (\%) of Thymus vulgaris L. plant as affected by the combination between foliar extract seaweed (Sw) and chitosan $(\mathrm{Ch})$ in the $2^{\text {nd }}$ cut at the second season (2018/2019). 
Table 6. Effect of seaweed extract, chitosan and potassium silicate on photosynthetic pigment contents of Thymus vulgaris $L$. for two cuts during 2017/2018 and 2018/2019 seasons.

\begin{tabular}{|c|c|c|c|c|c|c|c|c|}
\hline \multirow{2}{*}{ Treatments } & \multicolumn{2}{|c|}{$\begin{array}{c}\text { Chl. a } \\
\text { (mg/g F.W.) }\end{array}$} & \multicolumn{2}{|c|}{$\begin{array}{c}\text { Chl. b } \\
\text { (mg/g F.W.) }\end{array}$} & \multicolumn{2}{|c|}{$\begin{array}{c}\text { Carot. } \\
\text { (mg/g F.W.) }\end{array}$} & \multicolumn{2}{|c|}{ Total chl } \\
\hline & $1^{\text {st }}$ cut & $2^{\text {nd }}$ cut & $1^{\text {st }}$ cut & $2^{\text {nd }}$ cut & $1^{\text {st }}$ cut & $2^{\text {nd }}$ cut & $1^{\text {st }}$ cut & $2^{\text {nd }}$ cut \\
\hline & \multicolumn{8}{|c|}{ First season $(2017 / 2018)$} \\
\hline Control & 1.118 & 1.125 & 0.225 & 0.288 & 1.112 & 1.133 & 1.343 & 1.413 \\
\hline Sw $2 \mathrm{ml} / \mathrm{l}$ & 1.155 & 1.224 & 0.319 & 0.324 & 1.285 & 1.318 & 1.474 & 1.548 \\
\hline Sw 4 ml/l & 1.273 & 1.351 & 0.410 & 0.471 & 1.348 & 1.392 & 1.683 & 1.822 \\
\hline Sw $6 \mathrm{ml} / \mathrm{l}$ & 1.325 & 1.498 & 0.478 & 0.493 & 1.537 & 1.692 & 1.803 & 1.991 \\
\hline Ch $2 \mathrm{ml} / \mathrm{l}$ & 1.125 & 1.148 & 0.341 & 0.347 & 1.144 & 1.204 & 1.446 & 1.494 \\
\hline Ch 4 ml/l & 1.231 & 1.338 & 0.364 & 0.428 & 1.335 & 1.344 & 1.595 & 1.766 \\
\hline Ch 6 ml/l & 1.288 & 1.417 & 0.441 & 0.471 & 1.447 & 1.469 & 1.729 & 1.888 \\
\hline Ksi 6 ml/l & 1.138 & 1.204 & 0.334 & 0.304 & 1.193 & 1.212 & 1.472 & 1.508 \\
\hline Ksi 9 ml/l & 1.257 & 1.349 & 0.370 & 0.396 & 1.263 & 1.358 & 1.627 & 1.745 \\
\hline Ksi $12 \mathrm{ml} / \mathbf{l}$ & 1.291 & 1.436 & 0.456 & 0.483 & 1.463 & 1.675 & 1.747 & 1.919 \\
\hline \multirow[t]{2}{*}{ L.S.D. at 5\% } & 0.205 & 0.371 & 0.253 & 0.205 & 0.423 & 0.549 & 0.460 & 0.574 \\
\hline & \multicolumn{8}{|c|}{ Second season $(2018 / 2019)$} \\
\hline Control & 1.258 & 1.364 & 0.371 & 0.406 & 1.343 & 1.348 & 1.629 & 1.770 \\
\hline Sw $2 \mathrm{ml} / \mathbf{l}$ & 1.371 & 1.391 & 0.348 & 0.349 & 1.584 & 1.614 & 1.719 & 1.740 \\
\hline Sw 4 ml/l & 1.458 & 1.588 & 0.452 & 0.479 & 1.666 & 1.686 & 1.910 & 2.059 \\
\hline Sw 6 ml/l & 1.596 & 1.610 & 0.584 & 0.617 & 1.748 & 1.721 & 2.180 & 2.227 \\
\hline Ch $2 \mathrm{ml} / \mathrm{l}$ & 1.393 & 1.357 & 0.392 & 0.416 & 1.448 & 1.461 & 1.775 & 1.773 \\
\hline Ch 4 ml/l & 1.427 & 1.431 & 0.431 & 0.458 & 1.601 & 1.582 & 1.873 & 1.889 \\
\hline Ch $6 \mathrm{ml} / \mathrm{l}$ & 1.502 & 1.528 & 0.526 & 0.541 & 1.638 & 1.625 & 2.059 & 2.069 \\
\hline Ksi 6 ml/l & 1.314 & 1.415 & 0.382 & 0.389 & 1.537 & 1.583 & 1.706 & 1.804 \\
\hline Ksi 9 ml/l & 1.349 & 1.499 & 0.446 & 0.496 & 1.644 & 1.654 & 1.780 & 1.995 \\
\hline Ksi $12 \mathrm{ml} / \mathbf{l}$ & 1.547 & 1.545 & 0.557 & 0.593 & 1.729 & 1.707 & 2.073 & 2.138 \\
\hline L.S.D. at 5\% & 0.338 & 0.246 & 0.213 & 0.211 & 0.405 & 0.373 & 0.551 & 0.457 \\
\hline
\end{tabular}

Sw: seaweed extract, $C h$ : chitosan, Ksi: potassium silicate

chlorophyll in fresh leaves compared with control. The highest values of photosynthetic pigments were resulted from the application of seaweeds extract at $6 \mathrm{ml} / 1$, potassium silicate at $12 \mathrm{ml} / 1$ and chitosan at $6 \mathrm{ml} / 1$, orderly.

\section{b. Effect of combination treatments between seaweed extract and chitosan:}

Data presented in Table (7) showed Chlorophyll a, b, carotenoids and the total content of chlorophyll in fresh leaves of Thymus vulgaris, L. plant as affected by combination between seaweeds $6 \mathrm{~m} / 1+$ chitosan $4 \mathrm{~m} / 1$ treatments were with order $1.924-1.954,0.607-0.711,1.922-1.943$ and 2.531-2.665 in the first season and its two cuts and 2.032-2.307, 0.772-0.938, 1.9491.943 and 2.804-3.245 in the second one compared with control $(1.118-1.125,0.225-$
$0.288,1.112-1.133,1.343-1.413)$ and (1.258$1.364, \quad 0.371-0.406, \quad 1.343-1.348, \quad 1.629-$ $1.770)$, respectively.

2. $\mathbf{N}, \mathbf{P}, \mathrm{K}$ and total carbohydrates in the leaves:

\section{a. Effect of seaweed extract, chitosan and potassium silicate:}

Data presented in Table (8) indicated that the thyme plants sprayed with foliar seaweed extract at different levels increased the chemical components compared to control. Nitrogen, $\mathrm{P}$ and $\mathrm{K}$ percent contents and total carbohydrates were increased by increasing the concentration of seaweed extract $(6 \mathrm{ml} / \mathrm{l})$, potassium silicate up to $(12$ $\mathrm{m} / \mathrm{l})$ as well as chitosan $(6 \mathrm{~m} / \mathrm{l})$, orderly, nevertheless the control treatment gave the lowest values in the two seasons of study. 
Table 7. Effect of combination treatments between seaweed extract and chitosan on photosynthetic pigment contents of Thymus vulgaris L. for two cuts during 2017/2018 and 2018/2019 seasons.

\begin{tabular}{|c|c|c|c|c|c|c|c|c|}
\hline \multirow[t]{2}{*}{ Treatments } & \multicolumn{2}{|c|}{$\begin{array}{c}\text { Chl. a } \\
\text { (mg/g F.W.) }\end{array}$} & \multicolumn{2}{|c|}{$\begin{array}{c}\text { Chl. b } \\
\text { (mg/g F.W.) }\end{array}$} & \multicolumn{2}{|c|}{$\begin{array}{c}\text { Carot. } \\
\text { (mg/g F.W.) }\end{array}$} & \multicolumn{2}{|c|}{ Total Chl. } \\
\hline & $1^{\text {st }}$ cut & $2^{\text {nd }}$ cut & $1^{\text {st }}$ cut & $2^{\text {nd }}$ cut & $1^{\text {st }}$ cut & $2^{\text {nd }}$ cut & $1^{\text {st }}$ cut & $2^{\text {nd }}$ cut \\
\hline & \multicolumn{8}{|c|}{ First season $(2017 / 2018)$} \\
\hline Control & 1.118 & 1.125 & 0.225 & 0.288 & 1.112 & 1.133 & 1.343 & 1.413 \\
\hline $\mathrm{Sw} 2 \mathrm{ml} / \mathrm{l}+\mathrm{Ch} 2 \mathrm{ml} / \mathrm{l}$ & 1.219 & 1.505 & 0.451 & 0.433 & 1.421 & 1.692 & 1.671 & 1.938 \\
\hline $\mathrm{Sw} 2 \mathrm{ml} / \mathrm{l}+\mathrm{Ch} 4 \mathrm{ml} / \mathrm{l}$ & 1.299 & 1.592 & 0.476 & 0.501 & 1.548 & 1.657 & 1.775 & 2.093 \\
\hline $\mathrm{Sw} 2 \mathrm{ml} / \mathrm{l}+\mathrm{Ch} 6 \mathrm{ml} / \mathrm{l}$ & 1.353 & 1.609 & 0.481 & 0.506 & 1.697 & 1.698 & 1.834 & 2.110 \\
\hline $\mathrm{Sw} 4 \mathrm{ml} / \mathrm{l}+\mathrm{Ch} 2 \mathrm{ml} / \mathrm{l}$ & 1.387 & 1.673 & 0.459 & 0.519 & 1.658 & 1.702 & 1.846 & 2.192 \\
\hline $\mathrm{Sw} 4 \mathrm{ml} / \mathrm{l}+\mathrm{Ch} 4 \mathrm{ml} / \mathrm{l}$ & 1.748 & 1.718 & 0.524 & 0.582 & 1.727 & 1.751 & 2.272 & 2.300 \\
\hline Sw $4 \mathrm{ml} / \mathrm{l}+\mathrm{Ch} 6 \mathrm{ml} / \mathrm{l}$ & 1.851 & 1.783 & 0.583 & 0.617 & 1.751 & 1.784 & 2.434 & 2.400 \\
\hline Sw $6 \mathrm{ml} / \mathrm{l}+\mathrm{Ch} 2 \mathrm{ml} / \mathrm{l}$ & 1.553 & 1.771 & 0.493 & 0.528 & 1.683 & 1.715 & 2.046 & 2.299 \\
\hline Sw $6 \mathrm{ml} / \mathrm{l}+\mathrm{Ch} 4 \mathrm{ml} / \mathrm{l}$ & 1.924 & 1.954 & 0.607 & 0.711 & 1.922 & 1.943 & 2.531 & 2.665 \\
\hline Sw $6 \mathrm{ml} / \mathrm{l}+\mathrm{Ch} 6 \mathrm{ml} / \mathrm{l}$ & 1.864 & 1.898 & 0.637 & 0.630 & 1.867 & 1.905 & 2.501 & 2.528 \\
\hline \multirow[t]{2}{*}{ L.S.D. at 5\% } & 0.702 & 0.825 & 0.382 & 0.423 & 0.810 & 0.832 & 0.538 & 0.525 \\
\hline & \multicolumn{8}{|c|}{ Second season $(2018 / 2019)$} \\
\hline Control & 1.258 & 1.364 & 0.371 & 0.406 & 1.343 & 1.348 & 1.629 & 1.770 \\
\hline $\mathrm{Sw} 2 \mathrm{ml} / \mathrm{l}+\mathrm{Ch} 2 \mathrm{ml} / \mathrm{l}$ & 1.621 & 1.566 & 0.516 & 0.415 & 1.643 & 1.664 & 2.137 & 1.981 \\
\hline $\mathrm{Sw} 2 \mathrm{ml} / \mathrm{l}+\mathrm{Ch} 4 \mathrm{ml} / \mathrm{l}$ & 1.677 & 1.685 & 0.537 & 0.492 & 1.731 & 1.684 & 2.214 & 2.177 \\
\hline $\mathrm{Sw} 2 \mathrm{ml} / \mathrm{l}+\mathrm{Ch} 6 \mathrm{ml} / \mathrm{l}$ & 1.723 & 1.746 & 0.584 & 0.598 & 1.824 & 1.774 & 2.307 & 2.344 \\
\hline $\mathrm{Sw} 4 \mathrm{ml} / \mathrm{l}+\mathrm{Ch} 2 \mathrm{ml} / \mathrm{l}$ & 1.792 & 1.852 & 0.609 & 0.593 & 1.814 & 1.758 & 2.401 & 2.445 \\
\hline $\mathrm{Sw} 4 \mathrm{ml} / \mathrm{l}+\mathrm{Ch} 4 \mathrm{ml} / \mathrm{l}$ & 1.835 & 1.929 & 0.690 & 0.721 & 1.869 & 1.811 & 2.525 & 2.650 \\
\hline $\mathrm{Sw} 4 \mathrm{ml} / \mathrm{l}+\mathrm{Ch} 6 \mathrm{ml} / \mathrm{l}$ & 1.983 & 1.932 & 0.739 & 0.753 & 1.918 & 1.856 & 2.722 & 2.685 \\
\hline $\mathrm{Sw} 6 \mathrm{ml} / \mathrm{l}+\mathrm{Ch} 2 \mathrm{ml} / \mathrm{l}$ & 1.801 & 1.871 & 0.665 & 0.699 & 1.838 & 1.799 & 2.466 & 2.570 \\
\hline Sw $6 \mathrm{ml} / \mathrm{l}+\mathrm{Ch} 4 \mathrm{ml} / \mathrm{l}$ & 2.032 & 2.307 & 0.772 & 0.938 & 1.949 & 1.943 & 2.804 & 3.245 \\
\hline Sw 6 ml/l + Ch 6 ml/l & 2.055 & 1.975 & 0.743 & 0.924 & 1.936 & 1.876 & 2.798 & 2.899 \\
\hline L.S.D. at 5\% & 0.774 & 0.943 & 0.372 & 0.532 & 0.533 & 0.595 & 0.661 & 0.574 \\
\hline
\end{tabular}

Sw: seaweed extract, $\mathrm{Ch}$ : chitosan

\section{b. Effect of combination treatments} between seaweed extract and chitosan:

Data presented in Table (9) illustrated that leaves contents of $\mathrm{N}, \mathrm{P}, \mathrm{K} \%$ were greatly affected by all treatments compared with the control, in the two cuts of the two seasons of study. Combination between seaweeds $6 \mathrm{ml} / 1+$ chitosan $4 \mathrm{ml} / 1$ treatments recorded the following values for $\mathrm{N}, \mathrm{P}, \mathrm{K}$ and total carbohydrates in the first and second cuts of the first season (3.23-3.31, 0.45-0.48, 1.44-1.46, 38.43-40.55), respectively. Also, recorded the following values in the second season $(3.25-3.42,0.43$ $0.49,1.45-1.49,40.81-42.86)$, respectively.
While the control treatment recorded 1.14$1.18,0.12-0.14,1.02-1.06,30.05-32.06$ in the two cuts of the first season orderly and $1.21-1.25,0.17-0.15,1.06-1.09,31.10$ 34.12 in both cuts of the second season. The same trend of increasing $\mathrm{N}, \mathrm{P}$ and $\mathrm{K} \%$ due to seaweed extract potassium silicate was also reported by Zewail (2014). While, the efficiency of potassium silicate in promoting $\mathrm{N}, \mathrm{P}$ and $\mathrm{K} \%$ was revealed. Moreover, previous studies have shown the involvement of chitosan in increasing $\mathrm{N}, \mathrm{P}$ and $\mathrm{K} \%$ such as Saif Eldeen et al. (2014) on globe artichoke. 
Table 8. Effect of seaweed extract, chitosan and potassium silicate on nitrogen, phosphorus, potassium and total carbohydrates (\% of D.W.) of Thymus vulgaris $\mathrm{L}$. for two cuts during $2017 / 2018$ and $2018 / 2019$ seasons.

\begin{tabular}{|c|c|c|c|c|c|c|c|c|}
\hline \multirow[t]{2}{*}{ Treatments } & \multicolumn{2}{|c|}{$\mathbf{N} \%$} & \multicolumn{2}{|c|}{$\mathbf{P} \%$} & \multicolumn{2}{|c|}{$K \%$} & \multicolumn{2}{|c|}{$\begin{array}{c}\text { Total carbohydrates } \\
(\%)\end{array}$} \\
\hline & $1^{\text {st }}$ cut & $2^{\text {nd }}$ cut & $1^{\text {st }}$ cut & $2^{\text {nd }}$ cut & $1^{\text {st }}$ cut & $2^{\text {nd }}$ cut & $1^{\text {st }}$ cut & $2^{\text {nd }}$ cut \\
\hline & \multicolumn{8}{|c|}{ First season $(2017 / 2018)$} \\
\hline Control & 1.14 & 1.18 & 0.12 & 0.14 & 1.02 & 1.06 & 30.05 & 32.06 \\
\hline Sw $2 \mathrm{ml} / \mathrm{l}$ & 1.52 & 1.64 & 0.25 & 0.27 & 1.15 & 1.16 & 32.17 & 33.20 \\
\hline Sw $4 \mathrm{ml} / \mathrm{l}$ & 1.80 & 1.85 & 0.28 & 0.29 & 1.22 & 1.24 & 35.88 & 36.72 \\
\hline Sw $6 \mathrm{ml} / \mathrm{l}$ & 2.25 & 2.31 & 0.31 & 0.33 & 1.24 & 1.26 & 37.14 & 38.18 \\
\hline Ch $2 \mathrm{ml} / \mathrm{l}$ & 1.28 & 1.33 & 0.19 & 0.21 & 1.10 & 1.14 & 31.14 & 33.12 \\
\hline Ch $4 \mathrm{ml} / \mathrm{l}$ & 1.61 & 1.68 & 0.22 & 0.25 & 1.18 & 1.20 & 32.26 & 34.21 \\
\hline Ch $6 \mathrm{ml} / \mathrm{l}$ & 2.09 & 2.16 & 0.27 & 0.29 & 1.19 & 1.24 & 34.78 & 34.39 \\
\hline Ksi 6 ml/l & 1.36 & 1.42 & 0.21 & 0.26 & 1.12 & 1.16 & 32.15 & 33.18 \\
\hline Ksi 9 ml/l & 1.75 & 1.88 & 0.27 & 0.28 & 1.20 & 1.22 & 34.82 & 35.67 \\
\hline Ksi 12 ml/l & 2.11 & 2.21 & 0.29 & 0.31 & 1.23 & 1.24 & 35.12 & 36.14 \\
\hline \multirow[t]{2}{*}{ L.S.D. at 5\% } & 0.380 & 0.460 & 0.190 & 0.140 & 0.220 & 0.200 & 1.235 & 1.301 \\
\hline & \multicolumn{8}{|c|}{ Second season $(2018 / 2019)$} \\
\hline Control & 1.21 & 1.25 & 0.17 & 0.15 & 1.06 & 1.09 & 31.10 & 34.12 \\
\hline Sw $2 \mathrm{ml} / \mathrm{l}$ & 1.57 & 1.72 & 0.23 & 0.28 & 1.18 & 1.19 & 33.18 & 34.36 \\
\hline Sw $4 \mathrm{ml} / \mathrm{l}$ & 1.82 & 1.83 & 0.26 & 0.32 & 1.23 & 1.25 & 34.86 & 37.88 \\
\hline Sw $6 \mathrm{ml} / \mathrm{l}$ & 2.29 & 2.42 & 0.32 & 0.34 & 1.27 & 1.29 & 37.95 & 40.16 \\
\hline Ch $2 \mathrm{ml} / \mathrm{l}$ & 1.25 & 1.46 & 0.20 & 0.26 & 1.17 & 1.16 & 31.64 & 34.22 \\
\hline Ch $4 \mathrm{ml} / \mathrm{l}$ & 1.68 & 1.74 & 0.23 & 0.27 & 1.21 & 1.22 & 33.82 & 35.53 \\
\hline Ch 6 ml/l & 2.13 & 2.20 & 0.25 & 0.30 & 1.26 & 1.25 & 34.64 & 36.86 \\
\hline Ksi 6 ml/l & 1.41 & 1.59 & 0.24 & 0.29 & 1.16 & 1.18 & 33.15 & 35.28 \\
\hline Ksi 9 ml/l & 1.79 & 1.93 & 0.26 & 0.31 & 1.21 & 1.23 & 35.79 & 37.75 \\
\hline Ksi 12 ml/l & 2.17 & 2.35 & 0.30 & 0.33 & 1.25 & 1.27 & 36.91 & 38.00 \\
\hline L.S.D. at 5\% & 0.600 & 0.580 & 0.170 & 0.180 & 0.210 & 0.130 & 1.357 & 1.544 \\
\hline
\end{tabular}

Sw: seaweed extract, $\mathrm{Ch}$ : chitosan, Ksi: potassium silicate

\section{CONCLUSION}

From our results it could be concluded that the application of seaweed extract and chitosan on thyme had positive effects separately or combining together and significantly increased the growth, yield and percentage of essential oil. The best result gained from using at the highest concentrations of seaweed extract and chitosan (seaweed extract $6 \mathrm{ml} / \mathrm{l}$ water + chitosan $4 \mathrm{ml} / 1$ water).

\section{REFERENCES}

Abou-Baker, A.H.; Abd-Eladl, M. and Abbas, M.M. (2011). Use of silicate and different cultivation practices in alleviating salt stress effect on bean plants. Australian Journal of Basic and Applied Science, 5(9):769-781.

Atteya, A.K.G. and Amer, H.M. (2018). Influence of seaweed extract and amino acids on growth, productivity and chemical constituents of (Hibiscus sabdariffa L.) plants. Biosci. Res., 15(2):772-791.

Battacharyya, D.; Babgohari, M.Z.; Rathor, P., and Prithiviraj, B. (2015). Seaweed extracts as biostimulants in horticulture, Review. Scientia Horticulturae, 196:3948. 
Table 9. Effect of combination treatments between seaweed extract chitosan on nitrogen, phosphorus, potassium and total carbohydrates (\% of D.W.) of Thymus vulgaris $\mathrm{L}$. for two cuts during $2017 / 2018$ and 2018/2019 seasons.

\begin{tabular}{|c|c|c|c|c|c|c|c|c|}
\hline \multirow[t]{2}{*}{ Treatments } & \multicolumn{2}{|c|}{$\mathbf{N} \%$} & \multicolumn{2}{|c|}{$\mathbf{P \%}$} & \multicolumn{2}{|c|}{$\mathbf{K} \%$} & \multicolumn{2}{|c|}{$\begin{array}{c}\text { Total carbohydrates } \\
(\%)\end{array}$} \\
\hline & $1^{\text {st }}$ cut & $2^{\text {nd }}$ cut & $1^{\text {st }}$ cut & $2^{\text {nd }}$ cut & $1^{\text {st }}$ cut & $2^{\text {nd }}$ cut & $1^{\text {st }}$ cut & $2^{\text {nd }}$ cut \\
\hline & \multicolumn{8}{|c|}{ First season $(2017 / 2018)$} \\
\hline Control & 1.14 & 1.18 & 0.12 & 0.14 & 1.02 & 1.06 & 30.05 & 32.06 \\
\hline $\mathrm{Sw} 2 \mathrm{ml} / \mathrm{l}+\mathrm{Ch} 2 \mathrm{ml} / \mathrm{l}$ & 1.89 & 1.95 & 0.24 & 0.25 & 1.24 & 1.23 & 31.12 & 33.21 \\
\hline $\mathrm{Sw} 2 \mathrm{ml} / \mathrm{l}+\mathrm{Ch} 4 \mathrm{ml} / \mathrm{l}$ & 2.29 & 2.34 & 0.28 & 0.29 & 1.29 & 1.30 & 34.17 & 34.64 \\
\hline $\mathrm{Sw} 2 \mathrm{ml} / \mathrm{l}+\mathrm{Ch} 6 \mathrm{ml} / \mathrm{l}$ & 2.36 & 2.41 & 0.32 & 0.34 & 1.31 & 1.32 & 35.21 & 35.77 \\
\hline $\mathrm{Sw} 4 \mathrm{ml} / \mathrm{l}+\mathrm{Ch} 2 \mathrm{ml} / \mathrm{l}$ & 2.59 & 2.66 & 0.34 & 0.36 & 1.34 & 1.35 & 35.18 & 36.80 \\
\hline $\mathrm{Sw} 4 \mathrm{ml} / \mathrm{l}+\mathrm{Ch} 4 \mathrm{ml} / \mathrm{l}$ & 2.82 & 2.77 & 0.41 & 0.42 & 1.38 & 1.39 & 36.25 & 38.78 \\
\hline Sw 4 ml/l + Ch 6 ml/l & 2.86 & 2.84 & 0.42 & 0.43 & 1.40 & 1.42 & 38.29 & 39.45 \\
\hline Sw $6 \mathrm{ml} / \mathrm{l}+\mathrm{Ch} 2 \mathrm{ml} / \mathrm{l}$ & 2.75 & 2.79 & 0.39 & 0.45 & 1.36 & 1.38 & 35.22 & 36.86 \\
\hline Sw $6 \mathrm{ml} / \mathrm{l}+\mathrm{Ch} 4 \mathrm{ml} / \mathrm{l}$ & 3.23 & 3.31 & 0.45 & 0.48 & 1.44 & 1.46 & 38.43 & 40.55 \\
\hline Sw $6 \mathrm{ml} / \mathrm{l}+\mathrm{Ch} 6 \mathrm{ml} / \mathrm{l}$ & 3.21 & 3.11 & 0.42 & 0.47 & 1.41 & 1.44 & 39.31 & 39.51 \\
\hline \multirow[t]{2}{*}{ L.S.D. at 5\% } & 0.750 & 0.770 & 0.330 & 0.340 & 0.390 & 0.380 & 1.471 & 1.553 \\
\hline & \multicolumn{8}{|c|}{ Second season $(2018 / 2019)$} \\
\hline Control & 1.21 & 1.25 & 0.17 & 0.15 & 1.06 & 1.09 & 31.10 & 34.12 \\
\hline Sw $2 \mathrm{ml} / \mathrm{l}+\mathrm{Ch} 2 \mathrm{ml} / \mathrm{l}$ & 1.93 & 1.98 & 0.27 & 0.29 & 1.26 & 1.29 & 33.42 & 34.68 \\
\hline $\mathrm{Sw} 2 \mathrm{ml} / \mathrm{l}+\mathrm{Ch} 4 \mathrm{ml} / \mathrm{l}$ & 2.31 & 2.46 & 0.28 & 0.31 & 1.28 & 1.31 & 34.53 & 35.37 \\
\hline $\mathrm{Sw} 2 \mathrm{ml} / \mathrm{l}+\mathrm{Ch} 6 \mathrm{ml} / \mathrm{l}$ & 2.34 & 2.57 & 0.31 & 0.36 & 1.34 & 1.37 & 34.61 & 35.86 \\
\hline $\mathrm{Sw} 4 \mathrm{ml} / \mathrm{l}+\mathrm{Ch} 2 \mathrm{ml} / \mathrm{l}$ & 2.61 & 2.71 & 0.37 & 0.39 & 1.36 & 1.39 & 36.10 & 37.99 \\
\hline $\mathrm{Sw} 4 \mathrm{ml} / \mathrm{l}+\mathrm{Ch} 4 \mathrm{ml} / \mathrm{l}$ & 2.84 & 2.95 & 0.44 & 0.47 & 1.40 & 1.42 & 37.00 & 38.82 \\
\hline $\mathrm{Sw} 4 \mathrm{ml} / \mathrm{l}+\mathrm{Ch} 6 \mathrm{ml} / \mathrm{l}$ & 2.86 & 2.91 & 0.46 & 0.49 & 1.41 & 1.46 & 38.60 & 39.97 \\
\hline Sw $6 \mathrm{ml} / \mathrm{l}+\mathrm{Ch} 2 \mathrm{ml} / \mathrm{l}$ & 2.80 & 2.88 & 0.48 & 0.39 & 1.38 & 1.39 & 36.45 & 37.56 \\
\hline $\mathrm{Sw} 6 \mathrm{ml} / \mathrm{l}+\mathrm{Ch} 4 \mathrm{ml} / \mathrm{l}$ & 3.25 & 3.42 & 0.43 & 0.49 & 1.45 & 1.49 & 40.81 & 42.86 \\
\hline Sw $6 \mathrm{ml} / \mathrm{l}+\mathrm{Ch} 6 \mathrm{ml} / \mathrm{l}$ & 3.22 & 2.98 & 0.40 & 0.48 & 1.44 & 1.47 & 39.21 & 41.79 \\
\hline L.S.D. at $5 \%$ & 0.720 & 0.730 & 0.230 & 0.330 & 0.380 & 0.386 & 1.729 & 1.852 \\
\hline
\end{tabular}

Sw: seaweed extract, $C h$ : chitosan

Bistgani, Z.F.; Siadat, S.A.; Bakhshandeh, A.; Pirbaluti, A.G. and Hashemi, M. (2016). Influence of chitosan concentration on morpho- physiological traits, essential oil and phenolic content under different fertilizers application in Thymus daenensis. Journal of Herbal Drugs, 7(2):117-125.

British Pharmacopoeia (2000). The Pharmaceutical Press 17 Bloomsburg, Square. W.C.I. London.

Castro, G.T.; Avelelas, F.P. and Leandro, S. (2016). Chitosan Seed Soaking: Germination and Growth of Coriandrum sativum and Solanum lycopersicum. Proc. the International Meeting on Marine Research, Peniche, Portugal.
Delucia, B. and Vecchietti, L. (2012). Type of bio stimulant and application method effects on stem quality and root system growth in L.A. Lily. Europ. J. Hort. Sci., 77(1):10-15.

El-Leithy, A.S.; El-Attar, A.B.E.; ElGhadban, E.A.E. and Abdel-Ghani, N.M. (2019). Effect of salinity, seaweed extract and salicylic acid on growth, essential oil and chemical composition of Plectranthus amboinicus (Lour.). Journal Bioscience Research, 16(1):118-133.

Ezz El-Din, A.A.; Hendawy, S.F.; Aziz, E.E. and Omer, E.A. (2010). Enhancing growth, yield and essential oil of caraway plants by nitrogen and potassium 
fertilizers. International Journal of Academic Research, 2(3):192-197.

Gharib, F.; Zeid, I.; Salem, O. and Ahmed, E. (2014). Effects of (Sargassum latifolium) extract on growth, oil content and enzymatic activities of rosemary plants under salinity stress. Life Sci. J., 11(10):933-945.

Gomez, K.A. and Gomez, A.A. (1984). Statistical Procedures for Agricultural Research, $2^{\text {nd }}$ Ed. John Willey and Sons, New York, USA, 680 p.

Guenther, Z. and Joseph, S. (1978). Handbook Series in Chromatography. CRC Press Inc., USA.

Hadrami, A.E.; Adam, L.R.; Hadrami, I.E. and Daayf, F. (2010). Chitosan in plant protection. Mar. Drugs., 8:968-987.

Hadwiger, L.A. (2013). Multiple effects of chitosan on plant systems: solid science or hype. Plant Sci., 20(8):42-49.

Helaly, M.N.; Farouk, S.; Arafa, S.A. and Amhimmid, N. B. (2018). Inducing salinity tolerance of rosemary (Rosmarinus officinalis L.) plants by chitosan or zeolite application. Asian Journal of Advances in Agricultural Research, 5(4):1-20.

Herbert, D.; Phipps, P.J. and Strange, R.E. (1971). Chemical analysis of microbial cells. In: Norris, J.R. and Ribbons, D.W. (eds), Methods in Microbiology, Academic Press, USA, 5B:209-344.

Hoftman, E. (1967). Chromatography, $2^{\text {nd }} E d$. Reinhold Pulb. Corp., USA, pp. 208-515.

Hussien, A.A.; Ismail, A.A. and Sabra, F.S. (2011). Bioactive substances extracted from seaweeds as a biocontrol agent's effects and identification. Journal of Agriculture Research, Kafr El-Sheikh Univ., 37(1):183-208.

Jackson, M.L. (1967). Soil Chemical Analysis. Prentice-Hall of India Pvt. Ltd., New Delhi, India, 498 p.

Jensen, E. (2004). Fact or fancy, from the organic broad caster. Mid-West Organic and Sustainable Education, 12(3):164170.

Kamal, A.M. and Ghanem, K.M. (2011). Response of cape gooseberry plants
(Physalis peruviana L.) to some organic amendments and foliar spray with chitosan. Journal of Plant Production, Mansoura Univ., 2(12):1741-1759.

Khalid, A. (2014). Influences of silicate dissolving bacteria and natural potassium on growth and essential oil of rue (Ruta graveolens L.) plant. Thai J. Agric. Sci, 47(1):31-36.

Kim, H.J.; Chen, F.; Wang, X. and Rajapakse, N. C. (2005). Effect of chitosan on the biological properties of sweet basil (Ocimum basilicum L.). Journal of Agriculture Food Chemistry, 53(9):696-701.

Mahdavi, B. (2013). Seed germination and growth responses of Isabgol (Plantago ovata Forsk.) to chitosan and salinity. Int. J. Agriculture and Crop Sciences, 5(10):1084-1088.

Mazumdar, B.C. and Majumder, K. (2003). Methods on Physic-Chemical Analysis of Fruits. Daya Publishing House, Delhi, India, $187 \mathrm{p}$.

Murphy, J.S. and Reilly, J.P. (1962). A modified single solution method for the determination of phosphate in natural water. Chemical Analysis, Acta Hort., 27:31-36.

Nasiroleslami, E. and Safaridolatabad, S. (2014). The comparison of organic and biologic fertilizers effects on growth and essential oil of dill (Anethum graveolens L.). Int. J. Biosci., 5(7):65-74.

Norrie, J. and Keathley, J. (2006). Benefit of Ascophyllumn odosummarin plant extracts application to Thompson seedless grape production. Acta Hort., 7(27):243-247.

Ordog, V.; Stirk, W.A.; VanStaden, J.; Novak, O. and Strand, M. (2004). Endogenous cytokinins in the three genera of microalgae from the chlorophyte. J. Physiol., 40:88-95.

Piper, O.S. (1947). Soil and Plant Analysis. Inter. Sci. Publishers. New York, USA, pp. 213-217.

Saif Eldeen, U.M.; Shokr, M.M.B. and El Shotoury, R.S. (2014). Effect of foliar spray with seaweeds extract and chitosan 


\section{A.A. Waly et al.}

on earliness and productivity of globe artichoke. Journal of Plant Production, Mansoura Univ., 5(7):1197-1207.

Salama, A.M. and Yousef, R.S. (2015). Response of basil plant (Ocimum sanctum L.) to foliar spray with amino acids or seaweed extract. J. Hort. Sci. Ornam. Plants, 7(3):94-106.

Shaaban, M.M. (2011). Green microalgae water extract as foliar feeding to wheat plants. Pakistan J. Biol. Sci., 4: 628-632.

Shabana, Y.M.; Abdalla, M.E.; Hilal, A.A. and AbdelAziz, H.M. (2016). Performance of three silicon sources in suppressing Rhizoctonia solani diseases on sage (Salvia officinalis L.) and in improving yield of plant herb and essential oil. J. Plant Prot. Pathol., 7(9):555-563.

Smirnoff, N. (1995). Antioxidant systems and plant response to the environment. In: Smirnoff, N. (ed.), Environment and Plant Metabolism, Flexibility and Acclimation, Oxford: Bios Scientific Pub-lashers, pp. 217-243.

Wand, S.Y. and Galletta, G.J. (1998). Foliar application of potassium silicate induces metabolic changes in strawberry plants. J. Plant Nutr., 21(1):157-167.

Wang, M.; Zheng, Q.; Shen, Q. and Guo, S. (2013). The critical role of potassium in plant stress response. International Journal of Molecular Science, 14(4):7370-7390.

Wettestein, V.D. (1957). Chlorophyll-Lethal under submikroskopische, Formwechsel der Plastiden. Exp. Cell Res., 12(3):427506.

Wilde, S.A.; Corey, R.B.; Lyer, J.G. and Voigt, G.K. (1985). Soil and Plant Analysis for Tree Culture, $3^{\text {rd }}$ Ed. Oxford and IBM Publishing Co., New Delhi, India, pp. 529-546.

Zewail, R.M.Y. (2014). Effect of seaweed extract and amino acids on growth and productivity and some biocostituents of common bean (Phaseolus vulgaris L.) plants. Journal of Plant Production, Mansoura Univ., 5(8):1441-1453.

$$
\begin{aligned}
& \text { تعزيز النمو والانتاجية ونسبة الزيت العطري لنبات الزعتر باستخدام مستخلص الطحالب البحرية }
\end{aligned}
$$

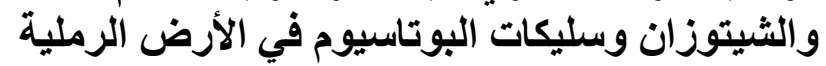

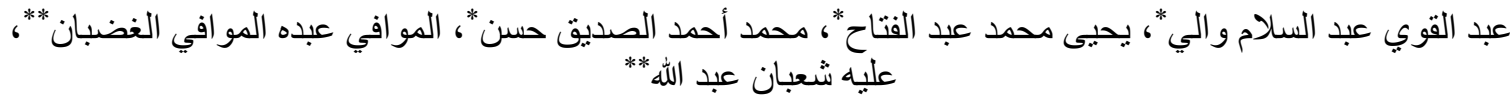

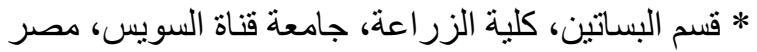

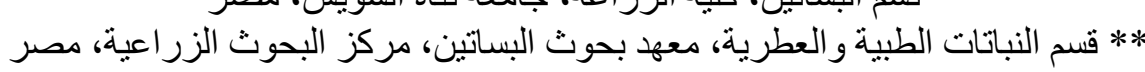

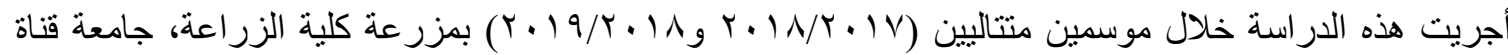

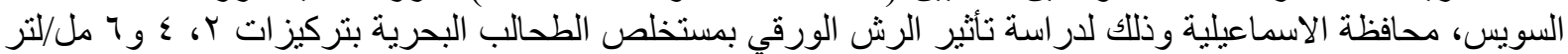

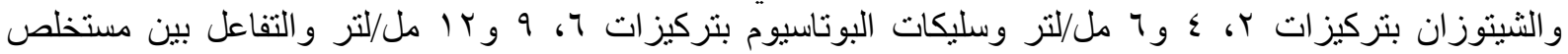

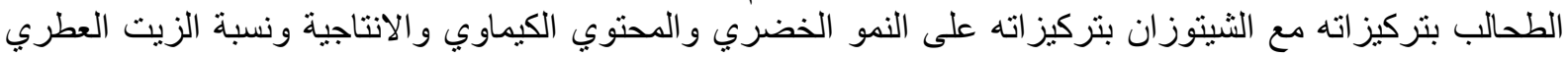

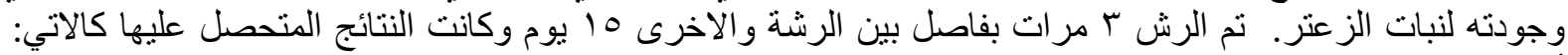

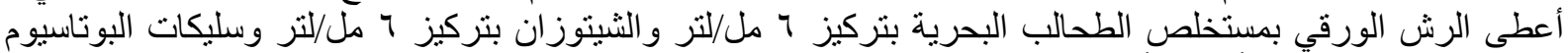

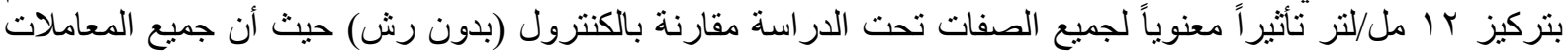

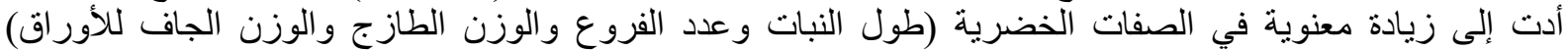

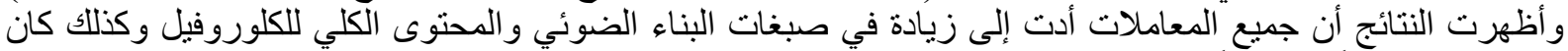

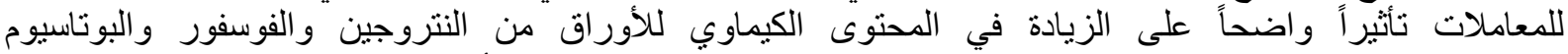

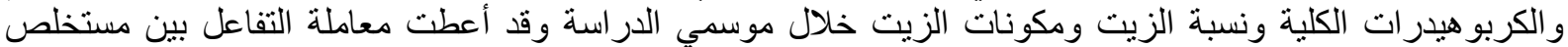

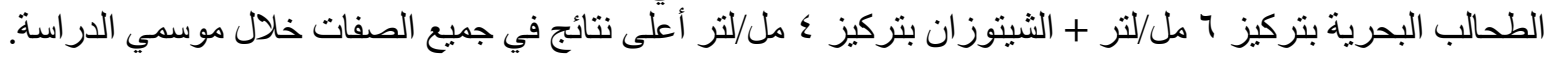

